\title{
Some Properties of Generalized Topologies in GTSs
}

\author{
S. Vadakasi ${ }^{1^{*}}$
}

\begin{abstract} topological space. Finally, we analyze the nature of some special spaces.

Keywords: Genralized topology, Baire space, Stack and p-stack.

2010 AMS: 54A10, 54A05.

${ }^{1}$ A.K.D.Dharma Raja Women's College, Rajapalayam, Tamilnadu

*Corresponding author: vadakasisub1992@gmail.com

Received: 15 July 2020, Accepted: 26 September 2020, Available online: 29 September 2020
\end{abstract}

In this article, we introduce one new generalized topology and investigate its properties in a generalized topological space. Also, we give various properties of some generalized topologies defined in a generalized

\section{Introduction}

The notion of a generalized topological space was introduced by Császár in [3]. Let $X$ be any non-null set. A family $\mu \subset \exp (X)$ is a generalized topology [9] in $X$ if $\emptyset \in \mu$ and $\bigcup_{t \in T} G_{t} \in \mu$ whenever $\left\{G_{t} \mid t \in T\right\} \subset \mu$ where $\exp (X)$ is a power set of $X$. We call the pair $(X, \mu)$ as a generalized topological space (GTS) [9]. If $X \in \mu$, then the pair $(X, \mu)$ is called a strong generalized topological space (sGTS) [9]. Let $Y \subset X$. Then the subspace generalized topology [2] is defined by, $\mu_{Y}=\{Y \cap U \mid U \in \mu\}$ and the pair $\left(Y, \mu_{Y}\right)$ is called as the subspace generalized topological space [2].

Let $(X, \mu)$ be a GTS and $A \subset X$. The interior of $A$ [9] denoted by $i A$, is the union of all $\mu$-open sets contained in $A$ and the closure of $A$ [9] denoted by $c A$, is the intersection of all $\mu$-closed sets containing $A$ when no confusion can arise. The elements in $\mu$ are called the $\mu$-open sets, the complement of a $\mu$-open set is called the $\mu$-closed set and the complement of $\mu$ is denoted by $\mu^{\prime}$. Denote $\{U \in \mu \mid U \neq \emptyset\}$ by $\tilde{\mu}$ [8] and denote $\{U \in \mu \mid x \in U\}$ by $\mu(x)$ [8].

Throughout this paper, $\mathbb{R}, \mathbb{Z}, \mathbb{Q}$ and $\mathbb{N}$ denote the set of all real numbers, integers, rational numbers and natural numbers, respectively. The notations $X_{3}, X_{4}, X_{5}$ and $X_{6}$ are mean the sets $\{a, b, c\},\{a, b, c, d\},\{a, b, c, d, e\}$ and $\{a, b, c, d, e, f\}$, respectively.

\section{Preliminaries}

In this section, we remember some basic definitions and lemmas which will be useful in the development of the next sections.

A subset $A$ of a GTS $(X, \mu)$ is said to be a $\mu$-nowhere dense [6] (resp. $\mu$-dense [6], $\mu$-codense [7]) set if icA $=\emptyset$ (resp. $c A=X, c(X-A)=X)$. $A$ is said to be a $\mu$-strongly nowhere dense set if for every $V \in \tilde{\mu}$, there is $U \in \tilde{\mu}$ such that $U \subset V$ and $U \cap A=\emptyset$. Then $A$ is said to be a $\mu$-meager (or $\mu$-first category) (resp. $\mu$-s-meager (or $\mu$-s-first category)) set [8] if $A=\bigcup_{n \in \mathbb{N}} A_{n}$ where $A_{n}$ is $\mu$-nowhere dense (resp. $\mu$-strongly nowhere dense) for all $n \in \mathbb{N}$.

In a GTS, every subset of a $\mu$-strongly nowhere dense set is $\mu$-nowhere dense and every subset of a $\mu$-meager (resp. $\mu$-s-meager) set is $\mu$-meager (resp. $\mu$-s-meager) [8].

Let $(X, \mu)$ be a GTS and $A \subset X$. Then $A$ is said to be a $\mu$-second category ( $\mu$-II category) (resp. $\mu$-s-second category ( $\mu$-s-II category)) set [8] if $A$ is not $\mu$-meager (resp. $\mu$-s-meager). $A$ is $\mu$-residual (resp. $\mu$-s-residual) [8] if $X-A$ is $\mu$-meager 
(resp. $\mu$-s-meager).

A GTS $(X, \mu)$ is said to be $\mu$-II category (resp. $\mu$-s-II category) if $X$ is $\mu$-II category (resp. $\mu$-s-II category) as a subset. A space $X$ is called a Baire space (BS) [8] if each $V \in \tilde{\mu}$ is of $\mu$-II category in $X$. A space $(X, \mu)$ is a strong Baire space (sBS) [8] if $V_{1} \cap V_{2} \cap \ldots \cap V_{n}$ is of $\mu$-II category set for all $V_{1}, V_{2}, \ldots, V_{n} \in \mu$ such that $V_{1} \cap V_{2} \cap \ldots \cap V_{n} \neq \emptyset$. Also, every sBS is a BS [8].

Define $\mu^{\star}=\left\{\bigcup_{t}\left(U_{1}^{t} \cap U_{2}^{t} \cap U_{3}^{t} \cap \ldots \cap U_{n_{t}}^{t}\right) \mid U_{1}^{t}, U_{2}^{t}, \ldots, U_{n_{t}}^{t} \in \mu\right\}$ and $\mu^{\star \star}=\{A \subset X \mid A$ is of $\mu$-II category set $\} \cup\{\emptyset\}$ [8]. Then $\mu \subset \mu^{\star}$ and $\mu \subset \mu^{\star \star}$ if $(X, \mu)$ is a Baire space [8]. Also, $\mu^{\star} \subset \mu^{\star \star}$ if $(X, \mu)$ is a sBS [11].

A space $(X, \mu)$ is called hyperconnected [6] if every non-null $\mu$-open subset of $X$ is $\mu$-dense in $X$. A GTS $(X, \mu)$ is said to be a generalized submaximal space [7] if every $\mu$-dense subset of $X$ is a $\mu$-open set in $X$.

The following lemmas will be useful in the sequel.

Lemma 2.1. [8, Property 2.3] Let $(X, \mu)$ be a GTS and $A \subset X$ be a $\mu$-nowhere (resp. $\mu$-strongly nowhere) dense set. Then the closure of $A$ and any subset of $A$ are $\mu$-nowhere (resp. $\mu$-strongly nowhere) dense sets.

Lemma 2.2. [8, Property 2.5] Let $(X, \mu)$ be a GTS and $A \subset X$. Then the following hold.

(a) If $A$ is s-meager then it is meager.

(b) If $A$ is of II category then it is of s-II category.

(c) If $A$ is s-residual then it is residual.

Lemma 2.3. [9, Proposition 4.7] Let $(X, \mu)$ be a GTS. If $F_{n}$ is a $\mu$-meager set for each $n \in \mathbb{N}$, then $\bigcup_{n \in \mathbb{N}} F_{n}$ is a $\mu$-meager set in $X$.

Lemma 2.4. [9, Theorem 5.3] Let $(X, \mu)$ be a GTS. The following are equivalent.

(a) $X$ is Baire.

(b) If $A \neq \emptyset$ is $\mu$-residual in $X$, then $A$ is $\mu$-dense in $X$.

(c) If $B \neq X$ is $\mu$-meager in $X$, then $B$ is $\mu$-codense in $X$.

(d) Every $U \in \tilde{\mu}$ is $\mu$-II category in $X$.

(e) $i F=\emptyset$, for every $F$ is a $\mu$-meager set in $X$.

(f) For every $\mu$-closed set $F_{n}$ with $i F_{n}=\emptyset, i\left(\bigcup_{n \in \mathbb{N}} F_{n}\right)=\emptyset$.

Lemma 2.5. [12, Theorem 3.3] Let $(X, \mu)$ be a GTS. Then the following hold.

(a) If $G_{n}$ is $\mu$-s-meager for each $n \in \mathbb{N}$, then $\bigcup_{n \in \mathbb{N}} G_{n}$ is $\mu$-s-meager.

(b) If $F_{n}$ is $\mu$-s-residual for each $n \in \mathbb{N}$, then $\bigcap_{n \in \mathbb{N}} F_{n}$ is $\mu$-s-residual.

\section{Properties of Generalized Topology}

In this section, we give some properties of generalized topologies defined in a generalized topological space. Also, we check some families are either satisfied with the stack property or not.

We start the study of various types of generalized topologies in a generalized topological space by reminding the well-known definitions in GTSs.

Let $(X, \mu)$ be a GTS. A collection $\mathscr{C}$ of subsets of $X$ is called a stack [10] if $A \in \mathscr{C}$ whenever $B \in \mathscr{C}$ and $B \subset A$. A stack $\mathscr{H}$ on $X$ is called a $p$-stack [10] if $A, B \in \mathscr{H}$, then $A \cap B \neq \emptyset$.

Theorem 3.1. Let $(X, \mu)$ be a GTS. Then $\tilde{\mu}^{\star \star}$ is a stack.

Proof. Suppose $B \in \tilde{\mu}^{\star \star}$ and $B \subset A \subset X$. Then $B$ is of $\mu$-II category set in $X$. Since subset of a $\mu$-meager set is $\mu$-meager, $A$ is of $\mu$-II category set in $X$ implies that $A \in \tilde{\mu}^{\star \star}$. Therefore, $\tilde{\mu}^{\star \star}$ is a stack.

The below Corollary 3.2 directly follows from Theorem 3.1 so the proof is omitted.

Corollary 3.2. Let $(X, \mu)$ be a GTS and $A \subset X$. Then the following hold.

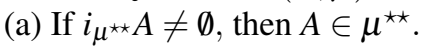

(b) If $(X, \mu)$ is a BS and if $i_{\mu} A \neq \emptyset$, then $A \in \mu^{\star \star}$.

(c) If $(X, \mu)$ is a sBS and if $i_{\mu^{\star}} A \neq \emptyset$, then $A \in \mu^{\star \star}$.

(d) If $A \in \mu^{\star \star}$, then $c_{\mu} A, c_{\mu} \star A, c_{\mu \star} A \in \mu^{\star \star}$.

The following Example 3.3, (a) shows that the generalized topology $\tilde{\mu}$ is not a stack in a generalized topological space, (b) proves that there exist a topology $\mu$, in which $\tilde{\mu}$ is not a stack and (c) proves that $\mu$ is not a topology even if $\tilde{\mu}$ is a stack. The generalized topology $\tilde{\mu}^{\star}$ is not a stack as shown by the below Example 3.4. 
Example 3.3. (a) Consider the generalized topological space $(X, \mu)$ where $X=\mathbb{R}$ and $\mu$ is the $\mathbb{Z}$ forbidden generalized topology on $\mathbb{R}$, that is, $\mu=\{U \subset \mathbb{R} \mid U \subset \mathbb{R}-\mathbb{Z}\}$. Then $\tilde{\mu}$ is not a stack. Because, if $U=\left\{\frac{n}{n+1} \mid n \in \mathbb{N}\right\}$, then $U \in \tilde{\mu}$. Here $U \subset \mathbb{Q}$. But $\mathbb{Q} \notin \tilde{\mu}$.

(b) Consider the generalized topological space $(X, \mu)$ where $X=[0,5]$ and $\mu=\{\emptyset,[0,2),(1,2),(1,4],[0,4], X\}$. Then $\mu$ is a topology. But $\tilde{\mu}$ is not a stack. For, let $G=[0,2)$ and $H=[0,2]$. Then $G \subset H$ and $G \in \tilde{\mu}$. But $H \notin \tilde{\mu}$.

(c) Consider the generalized topological space $\left(X_{4}, \mu\right)$ where $\mu=\left\{\emptyset,\{a, b\},\{a, b, c\},\{a, b, d\},\{a, c, d\},\{b, c, d\}, X_{4}\right\}$. Then $\tilde{\mu}$ is a stack. But $\mu$ is not a topology.

Example 3.4. Consider the generalized topological space $(X, \mu)$ where $X=[0,5]$ and $\mu=\left\{\emptyset,[0,2),\left(1, \frac{3}{2}\right),(1,4],[0,4]\right\}$. Then $\mu^{\star}=\left\{\emptyset,[0,2),(1,4],\left(1, \frac{3}{2}\right),(1,2),[0,4]\right\}$. Let $A=(1,2) \in \tilde{\mu}^{\star}$ and $B=[1,2]$. Then $A \subset B$. But $B \notin \tilde{\mu}^{\star}$. Thus, $\tilde{\mu}^{\star}$ is not a stack.

Theorem 3.5. Let $(X, \mu)$ be a GTS. Then $\left(X, \mu^{\star \star}\right)$ is a hyperconnected space if and only if $\tilde{\mu}^{\star \star}$ is a $p$-stack.

Proof. Suppose that $\left(X, \mu^{\star \star}\right)$ is a hyperconnected space. By Theorem 3.1, $\tilde{\mu}^{\star \star}$ is a stack. Let $A, B \in \tilde{\mu}^{\star \star}$. Then $A$ and $B$ are non-null $\mu^{\star \star}$-open sets and so $A$ and $B$ are $\mu^{\star \star}$-dense sets in $X$, by hypothesis. Therefore, $A \cap B \neq \emptyset$. Hence $\tilde{\mu}^{\star \star}$ is a p-stack. The reverse implication is directly follows from the definition of p-stack so the proof is omitted.

Example 3.6. Consider the generalized topological space $(X, \mu)$ where $X=[0,5]$ and $\mu=\{\emptyset,[0,2),(1,4],[0,4]\}$. Then $\mu^{\star \star}=\{\emptyset\} \cup\{A, B \subset X \mid A \in \exp ((1,2))-\{\emptyset\}, A \subset B\}$ and so $\left(X, \mu^{\star \star}\right)$ is not a hyperconnected space. Because, if we take $W=\left[0, \frac{3}{2}\right) \cup\left(\frac{3}{2}, 5\right]$, then $W \in \mu^{\star \star}$ and hence $\left\{\frac{3}{2}\right\}$ is a $\mu^{\star \star}$-closed set but $\left\{\frac{3}{2}\right\}$ is a non-null $\mu^{\star \star}$-open set in $X$. Let $U=[0,1] \cup\left\{\frac{3}{2}\right\}$ and $V=\left\{\frac{17}{10}\right\} \cup[2,5]$. Then $U, V \in \tilde{\mu}^{\star \star}$. But $U \cap V=\emptyset$. Thus, $\tilde{\mu}^{\star \star}$ is not a p-stack.

Theorem 3.7. Let $(X, \mu)$ be a BS. If every non-null $\mu$-open set is a $\mu$-residual set, then $(X, \mu)$ is a hyperconnected space.

Proof. Let $G \in \tilde{\mu}$. Then by hypothesis, $G$ is $\mu$-residual in $X$. By Lemma $2.4, G$ is a $\mu$-dense set in $X$. Hence $(X, \mu)$ is a hyperconnected space.

Theorem 3.8. Let $(X, \mu)$ be a BS and every non-null $\mu$-open set is $\mu$-residual in $X$. If $(X, \mu)$ is a generalized submaximal space, then the following hold.

(a) $\mu^{\star \star}=\mu$.

(b) $\tilde{\mu}$ is a stack.

Proof. It is enough to prove (a) only. Since $(X, \mu)$ is a BS we have $\mu \subset \mu^{\star \star}$. Let $B \in \mu^{\star \star}$. If $B=\emptyset$, then there is nothing to prove. Suppose $B \in \tilde{\mu}^{\star \star}$. Then $B$ is of $\mu$-II category set and so $B$ is not $\mu$-meager so that $B$ is not a $\mu$-nowhere dense set. Thus, $i_{\mu} c_{\mu} B \neq \emptyset$. Take $V=i_{\mu} c_{\mu} B$. Then $V \in \tilde{\mu}$. By hypothesis and Theorem 3.7, $(X, \mu)$ is a hyperconnected space so that $V$ is $\mu$-dense set in $X$. Then $B$ is $\mu$-dense set in $X$. Since $(X, \mu)$ is a generalized submaximal space, $B$ is a $\mu$-open set. Therefore, $B \in \tilde{\mu}$ so that $B \in \mu$. Thus, $\mu^{\star \star} \subset \mu$. Hence $\mu^{\star \star}=\mu$.

In Theorem 3.8, replace the condition “ $(X, \mu)$ be a BS" by the condition " $(X, \mu)$ be a sBS", we get $\mu=\mu^{\star}=\mu^{\star \star}$ and then $\tilde{\mu}^{\star}$ is a stack.

The following Example 3.9 shows that the necessary conditions are can not be dropped in Theorem 3.8.

Example 3.9. (a) Consider the generalized topological space $(X, \mu)$ where $X=[0,5]$ and $\mu=\left\{\emptyset,[0,2),\left(1, \frac{3}{2}\right),(1,3],[0,3]\right\}$. Then $(X, \mu)$ is a BS and every non-null $\mu$-open set is a $\mu$-residual set in $X$. Let $A=[1,4]$ be a subset of $X$. Then $c_{\mu} A=X$ and so $A$ is a $\mu$-dense subset of $X$. But $A \notin \mu$. Thus, $(X, \mu)$ is not a generalized submaximal space. Here $\mu^{\star \star}=\{\emptyset\} \cup\{A, B \subset X \mid$ $\left.A \in \exp \left(\left(1, \frac{3}{2}\right)\right)-\{\emptyset\}, A \subset B\right\}$. Choose $W=[1,2]$. Then $W \in \mu^{\star \star}$. But $W \notin \mu$. Hence $\mu^{\star \star} \nsubseteq \mu$.

(b) Consider the generalized topological space $\left(X_{5}, \mu\right)$ where $\mu=\{\emptyset,\{a, b\},\{a, c\},\{a, d\},\{b, c\},\{a, b, c\},\{a, b, d\},\{a, b, e\}$, $\left.\{a, c, d\},\{a, c, e\},\{a, d, e\},\{b, c, d\},\{b, c, e\},\{a, b, c, d\},\{a, b, c, e\},\{a, b, d, e\},\{a, c, d, e\},\{b, c, d, e\}, X_{5}\right\}$. Therefore, $\left(X_{5}, \mu\right)$ is a generalized submaximal space and every non-null $\mu$-open set is a $\mu$-residual set in $X_{5}$. But $\left(X_{5}, \mu\right)$ is not a BS. For, if we take $A=\{a, c, d\}$ is a subset of $X_{5}$. Now $i_{\mu} c_{\mu}(\{a\})=i_{\mu}(\{a\})=\{\emptyset\} ; i_{\mu} c_{\mu}(\{c\})=i_{\mu}(\{c\})=\{\emptyset\} ; i_{\mu} c_{\mu}(\{d\})=i_{\mu}(\{d\})=\{\emptyset\}$. Then $\{a, c, d\}$ is a $\mu$-meager set and so $A$ is not a $\mu$-II category set in $X_{5}$. Here, $\mu^{\star \star}=\{\emptyset\}$. Hence $\mu \nsubseteq \mu^{\star \star}$.

(c) Consider the generalized topological space $\left(X_{5}, \mu\right)$ where $\mu=\{\emptyset,\{a\},\{b\},\{a, b\},\{a, c\},\{a, b, c\},\{a, b, d\},\{a, b, e\},\{b, c, d\}$, $\left.\{a, b, c, d\},\{a, b, c, e\},\{a, b, d, e\}, X_{5}\right\}$. Then $\left(X_{5}, \mu\right)$ is a BS and generalized submaximal space. Let $A=\{a\}$ be a subset of $X_{5}$. Then $X_{5}-A=\{b, c, d, e\}$. Consider, $i_{\mu} c_{\mu}(\{b\})=i_{\mu}(\{b, d, e\})=\{b\} \neq \emptyset$. Thus, $\{b\}$ is of $\mu$-II category set in $X_{5}$. Therefore, $X_{5}-A$ is of $\mu$-II category set in $X_{5}$ so that $X_{5}-A$ is not a $\mu$-meager set which implies that $A$ is not a $\mu$-residual set in $X_{5}$. Thus, there is a non-null $\mu$-open set which is not a $\mu$-residual set in $X_{5}$. Here, $\mu^{\star \star}=\{\emptyset\} \cup\left\{A \subset X_{5} \mid\right.$ either $a \in A$ or $\left.b \in A\right\}$. Let $G=\{a, c, d\}$. Then $G \in \mu^{\star \star}$. But $G \notin \mu$. Hence $\mu^{\star \star} \nsubseteq \mu$.

Theorem 3.10. Every collection of all non-null $\mu$-residual sets in $X$ is a stack where $\mu$ is a generalized topology on $X$. 
Proof. Let $\eta=\{A \subset X \mid A$ is a non-null $\mu$-residual set $\}$. Suppose that $V \in \eta$ and $V \subset U$. Then $V$ is $\mu$-residual and so $X-V$ is $\mu$-meager in $X$. Since $V \subset U, X-U \subset X-V$ so that $X-U$ is a $\mu$-meager set in $X$, since subset of a meager set is meager. Thus, $U$ is $\mu$-residual in $X$. Therefore, $U \in \eta$. Hence $\eta$ is a stack.

Theorem 3.11. Let $(X, \mu)$ be a GTS and $\eta=\{\emptyset\} \cup\{A \subset X \mid A$ is non-null $\mu$-residual set $\}$. Then $\eta$ is a topology on $X$.

Proof. By Theorem 3.10, $\eta$ is closed under arbitrary union. Also, $\eta$ is closed under finite intersection, by Lemma 2.3. Let $A \subset X$ be a non-null $\mu$-residual set. Then $\emptyset=(X-X) \subset(X-A)$ and so $X$ is a non-null $\mu$-residual set. Thus, $X \in \eta$. Therefore, $\eta$ is a topology on $X$.

The below Theorem 3.12 (a) follows from the similar arguments in Theorem 3.10, Theorem 3.12 (b) follows from Lemma 2.5 (b) and the same considerations in Theorem 3.11 so the proof is omitted.

Theorem 3.12. Let $(X, \mu)$ be a GTS. Then the following hold.

(a) Every collection of all $\mu$-s-residual sets in $X$ is a stack.

(b) If $\eta=\{\emptyset\} \cup\{A \subset X \mid A$ is non-null $\mu$-s-residual set $\}$, then $\eta$ is a topology on $X$.

Theorem 3.13. Let $(X, \mu)$ be a GTS. Then every collection of all $\mu$-dense sets in $X$ is a stack.

Theorem 3.14. Let $(X, \mu)$ be a generalized submaximal space. Then every collection of all $\mu$-dense sets in $X$ is a p-stack.

Proof. Let $\eta=\{A \mid A$ is $\mu$-dense subset of $X\}$. By Theorem 3.13, $\eta$ is a stack. Let $G, H \in \eta$. Then $G$ and $H$ are $\mu$-dense sets in $X$. By hypothesis, $G$ and $H$ are non-null $\mu$-open sets in $X$ so that $G \cap H \neq \emptyset$. Hence $\eta$ is a p-stack.

The following Example 3.15 shows that the condition " $(X, \mu)$ be a generalized submaximal space" can not be dropped in Theorem 3.14. Also, this example shows that the collection of all $\mu$-codense sets in $X$ is not a stack.

Example 3.15. Consider the generalized topological space $\left(X_{4}, \mu\right)$ where $\mu=\left\{\emptyset,\{a, b\},\{b, c\},\{a, b, c\},\{b, c, d\}, X_{4}\right\}$. Here, $\{b\}$ is a $\mu$-dense set. But $\{b\} \notin \mu$. Thus, $\left(X_{4}, \mu\right)$ is not a generalized submaximal space. Take $\eta=\{A \mid A$ is $\mu$-dense subset of $\left.X_{4}\right\}$. Then $\eta=\left\{\{b\},\{a, b\},\{a, c\},\{b, c\},\{b, d\},\{a, b, c\},\{a, b, d\},\{a, c, d\},\{b, c, d\}, X_{4}\right\}$. Take $A=\{a, c\}$ and $B=\{b, d\}$. Then $A, B \in \eta$. But $A \cap B=\emptyset$.

Take $\zeta$ is the collection of all $\mu$-codense sets in $X$. Then $\zeta=\{\emptyset,\{a\} .\{b\},\{c\}$, $\left.\{d\},\{a, c\},\{a, d\},\{b, d\},\{c, d\},\{a, c, d\}, X_{4}\right\}$. Choose $A=\{b, d\}$ and $B=\{b, c, d\}$. Then $A \in \zeta$ and $A \subset B$. But $B \notin \zeta$. Thus, $\zeta$ is not a stack.

Next, Theorem 3.16 follows from Lemma 2.4 and Theorem 3.14 so the direct proof is omitted.

Theorem 3.16. Let $(X, \mu)$ be a BS. If $(X, \mu)$ be a generalized submaximal space, then $\eta=\{A \subset X \mid A$ is a non-null $\mu$-residual set\} is a p-stack.

The following Theorem 3.17 follows from the fact that "super set of a dense set is dense" and the trivial proof is omitted.

Theorem 3.17. Let $(X, \mu)$ be a GTS and $\eta=\{\emptyset\} \cup\{A \subset X \mid A$ is $\mu$-dense $\}$. Then $\eta$ is a strong generalized topology on $X$.

The collection $\eta$ defined on the above Theorem 3.17 is not closed under the finite intersection as shown by the following Example 3.18.

Example 3.18. Consider the generalized topological space $\left(X_{4}, \mu\right)$ where $\mu=\left\{\emptyset,\{a, b\},\{b, d\},\{a, b, c\},\{a, b, d\},\{b, c, d\}, X_{4}\right\}$. Take $\eta=\{\emptyset\} \cup\left\{A \subset X_{4} \mid A\right.$ is $\mu$-dense set $\}$. Then $\eta=\{\emptyset\} \cup\{\{b\},\{a, b\},\{a, d\},\{b, c\},\{b, d\},\{a, b, c\},\{a, b, d\},\{a, c, d\},\{b, c, d\}$, $\left.X_{4}\right\}$. Let $A=\{a, d\}$ and $B=\{b, d\}$. Then $A, B \in \eta$. But $A \cap B=\{d\} \notin \eta$. Thus, $\eta$ is not closed under the finite intersection.

Theorem 3.19. Let $(X, \mu)$ be a GTS. Then $\mu^{\star \star} \neq\{\emptyset\}$ if and only if $\mu^{\star \star}$ is a strong GT in $X$ where $\mu^{\star \star}=\left\{\bigcup_{t}\left(W_{1}^{t} \cap W_{2}^{t} \cap\right.\right.$ $\left.\left.\ldots \cap W_{n_{t}}^{t}\right) \mid W_{1}^{t}, W_{2}^{t}, \ldots, W_{n_{t}}^{t} \in \mu^{\star \star}\right\}$ and hence it is a topology.

Proof. Suppose that $\mu^{\star \star} \neq\{\emptyset\}$. Then there exists a non-null $\mu^{\star \star}$-open set in $X$. Take $G$ is the non-null $\mu^{\star \star}$-open set in $X$. Then $G$ is of $\mu$-II category set in $X$. Since subset of a $\mu$-meager set is $\mu$-meager, $X$ is of $\mu$-II category. Therefore, $X \in \mu^{\star \star}$ and so $X \in \mu^{\star \star^{\star}}$. Hence $\mu^{\star \star^{\star}}$ is a strong generalized topology. Also, $\mu^{\star \star^{\star}}$ is closed under finite intersection. Hence $\mu^{\star \star^{\star}}$ is a topology. Converse, follows from the definition of $\mu^{\star \star^{\star}}$.

Theorem 3.20. Let $(X, \mu)$ be a GTS. If $\tilde{\mu}$ is a stack, then the following hold.

(a) $\mu$ is a strong GT.

(b) $\mu^{\star}$ is a topology. 
Proof. It is enough to prove (a) only. Suppose $\mu \neq\{\emptyset\}$ and $\tilde{\mu}$ is a stack. Then we can choose a non-null open set in $\mu$. Take $G$ is a non-null $\mu$-open set in $X$. If $G=X$, then there is nothing to prove. Assume that, $G \subset X$. By hypothesis, $X \in \tilde{\mu}$ so that $X \in \mu$. Hence $\mu$ is a strong GT.

The converse implication of (a) in Theorem 3.20 is not true as shown by the following Example 3.21.

Example 3.21. Consider the generalized topological space $(X, \mu)$ where $X=[0,5]$ and $\mu=\{\emptyset,[0,2),(1,3],(1,5],[0,3], X\}$. Then $(X, \mu)$ is a strong generalized topological space. But $\tilde{\mu}$ is not a stack. For, $[0,2) \subset[0,2]$. Here $[0,2) \in \mu$ but $[0,2] \notin \mu$.

The following Theorem 3.22 is a direct consequence of the definition of the stack so the proof is omitted. If $\eta \subset \exp (X)-\{\emptyset\}$ where $X$ is a non-null set, $\mu \subset \eta \subset \gamma$ and if $\eta$ is a stack, then neither $\gamma$ nor $\mu$ is stack as shown by the following Example 3.23.

Theorem 3.22. Let $X$ be a non-null set and $\eta \subset \exp (X)$. If $\eta$ is a stack and generalized topology, then $\eta$ is a strong GT.

Example 3.23. Consider the non-null space $X_{4}$. Take $\eta=\left\{\{a\},\{a, b\},\{a, c\},\{a, d\},\{a, b, c\},\{a, b, d\},\{a, c, d\}, X_{4}\right\}$. Let $\gamma=$ $\left\{\{a\},\{b\},\{a, b\},\{a, c\},\{a, d\},\{a, b, c\},\{a, b, d\},\{a, c, d\}, X_{4}\right\}$ and $\mu=\left\{\{a\},\{a, b\},\{a, c\},\{a, b, c\},\{a, b, d\},\{a, c, d\}, X_{4}\right\}$. Then $\mu \subset \eta \subset \gamma$. Here $\eta$ is a stack. But neither $\gamma$ nor $\mu$ is stack. For, let $A_{1}=\{b\}, A_{2}=\{a\}$ and $B_{1}=\{b, d\}, B_{2}=\{a, d\}$. Then $A_{1} \in \gamma, A_{2} \in \mu$ and $A_{1} \subset B_{1}, A_{2} \subset B_{2}$. But $B_{1} \notin \gamma, B_{2} \notin \mu$.

Moreover, $\mu^{\star}$ is a topology if $\tilde{\mu}^{\star}$ is a stack.

\section{Nature of a New GT}

In this section, we define a new generalized topology and give some of its properties in a generalized topological space.

First of all, we recall some definitions and facts for the development of this section.

A GTS $(X, \mu)$ is said to be a weak Baire space (for short, wBS) [8] if for every $U \in \tilde{\mu}$ is of $\mu$-s-II category set in $X$. Also, every BS is a wBS.

Let $(X, \mu)$ be a GTS and $A \subset X$. Then $A$ is called $\mu$-semi-open (resp. $\mu$ - $\alpha$-open) if $A \subset$ ciA (resp. $A \subset i c i A$ ) [5].

In [8], Korczak - Kubiak et al. introduced a new generalized topology, namely $\mu^{\star \star}$, defined by using $\mu$-II category sets and gave some properties of this generalized topology in a generalized topological space.

Motivated by this, we will introduce a new generalized topology, namely $\mu^{\mathscr{V}}$, (dependent on GT $\mu$ ) in a generalized topological space which will be a convenient tool for considerations in this section.

The GT $\mu^{\mathscr{V}}$ defined as in the following way:

Definition 4.1. Let $(X, \mu)$ be a GTS. Then $\mu^{\mathscr{V}}=\{\emptyset\} \cup\{A \subset X \mid A$ is of $\mu$-s-II category set $\}$.

The family $\mu^{\mathscr{V}}$ is a strong generalized topology if $\mu^{\mathscr{V}} \neq\{\emptyset\}$. The converse implication is always true.

Let $(X, \mu)$ be a GTS. If $\mu \neq\{\emptyset\}$, then $X$ is of $\mu$-s-II category and hence $\mu^{\mathscr{V}}$ is a sGTS. Also, the reverse implication is true.

The following Example 4.2 shows that the family $\mu^{\mathscr{V}}$ is not closed under the finite intersection.

Example 4.2. Consider the generalized topological space $\left(X_{4}, \mu\right)$ where $\mu=\left\{\emptyset,\{a\},\{a, d\},\{b, d\},\{a, b, c\},\{a, b, d\}, X_{4}\right\}$. Then $\mu^{\mathscr{V}}=\{\emptyset\} \cup\left\{A \subset X_{4} \mid a \in A\right.$ or $b \in A$ or $\left.d \in A\right\}$. Here, $\{a, c\}$ and $\{b, c\}$ are of $\mu$-s-II category subsets in $X_{4}$. Take $A=\{a, c\} \cap\{b, c\}=\{c\}$. Then $A$ is a $\mu$-strongly nowhere dense set in $X_{4}$ and so $A$ is not $\mu$-s-II category in $X_{4}$ so that $A \notin \mu^{\mathscr{V}}$. Therefore, $\mu^{\mathscr{V}}$ is not closed under the finite intersection.

Lemma 4.3. [8, Lemma 2.12] Let $(X, \mu)$ be GTS and $A \subset X$. Then

$c_{\mu^{\star \star}}(A)=\left\{\begin{array}{c}X \text { if } A \text { is } \mu \text {-residual, } \\ A \text { if } A \text { is not } \mu \text {-residual }\end{array}\right.$

Lemma 4.4. [8, Lemma 2.13] Let $(X, \mu)$ be GTS, $X$ be a $\mu$-II category set and $A \subset X$. If $A$ is a $\mu^{\star \star}$-nowhere dense set, then $A$ is a $\mu$-meager set.

Theorem 4.5. Let $(X, \mu)$ be a GTS. Then $\tilde{\mu}{ }^{y}$ is a stack.

Proof. Suppose $A \subset B$ and $A \in \tilde{\mu^{\mathscr{V}}}$. Then $A$ is of $\mu$-s-II category set and so $B$ is of $\mu$-s-II category set, since subset of a $\mu$-s-meager set is $\mu$-s-meager. Therefore, $B \in \tilde{\mu^{\mathscr{V}}}$. Hence $\tilde{\mu^{\mathscr{V}}}$ is a stack.

The following Corollary 4.6 follows from the similar arguments in Theorem 3.5 and so the proof is omitted.

Corollary 4.6. Let $(X, \mu)$ be a GTS. Then $\tilde{\mu^{\mathscr{Y}}}$ is a p-stack if and only if $\left(X, \mu^{\mathscr{V}}\right)$ is a hyperconnected space. 
The following Proposition 4.7 directly follows from the definition of weak Baire space, strong Baire space and Lemma 2.2 (b) so the proof is omitted. Theorem 4.8 directly follows from Theorem 4.5, Proposition 4.7 so the trivial proof is omitted.

Proposition 4.7. Let $(X, \mu)$ be a GTS. Then the following hold.

(a) If $(X, \mu)$ is a wBS, then $\mu \subset \mu^{\mathscr{V}}$.

(b) If $(X, \mu)$ is a sBS, then $\mu^{\star} \subset \mu^{\mathscr{V}}$.

(c) $\mu^{\star \star} \subset \mu^{\mathscr{V}}$.

Theorem 4.8. Let $(X, \mu)$ be a GTS and $A \subset X$. Then the following hold.

(a) If $i_{\mu^{\mathscr{V}}} A \neq \emptyset$, then $A \in \mu^{\mathscr{V}}$.

(b) If $(X, \mu)$ is a wBS and if $i_{\mu} A \neq \emptyset$, then $A \in \mu^{\mathscr{V}}$.

(c) If $(X, \mu)$ is a sBS and if $i_{\mu^{\star}} A \neq \emptyset$, then $A \in \mu^{\mathscr{V}}$.

(d) If $A \in \mu^{\mathscr{V}}$, then $c_{\mu} A, c_{\mu^{\star}} A, c_{\mu^{\star}} A, c_{\mu^{\gamma}} A \in \mu^{\mathscr{V}}$.

Theorem 4.9. Let $(X, \mu)$ be a $w B S$-sGTS and $A \subset X$. Then the following hold.

(a) If $A$ is $\mu$ - $\alpha$-open, then $A \in \mu^{\mathscr{V}}$.

(b) If $A$ is $\mu$-semi-open, then $A \in \mu^{\mathscr{V}}$.

Proof. We will present the detailed proof only for (a). Suppose $A$ is a $\mu$ - $\alpha$-open set in $X$. Then $A \subset i c i A$. If $A=\emptyset$, then there is nothing to prove. Assume that, $A \neq \emptyset$. Then $i c i A \neq \emptyset$ so that $c i A \neq \emptyset$ which implies that $i A \neq \emptyset$, since $\mu$ is a sGT. Thus, $i_{\mu} A \neq \emptyset$. By hypothesis and Theorem 4.8 (b), $A \in \mu^{\mathscr{V}}$.

By using Theorem 4.9, immediately we get two Observations as follows.

Observation 4.10. Let $(X, \mu)$ be a $w B S$-sGTS. If $A$ is a $\mu^{\mathscr{V}}$-dense subset of $X$, then the following hold.

(a) $A \cap U \neq \emptyset$ for every non-null $\mu$ - $\alpha$-open set $U$.

(b) $A \cap V \neq \emptyset$ for every non-null $\mu$-semi-open set $V$.

Observation 4.11. Let $(X, \mu)$ be a $w B S$-sGTS and $A \subset X$. If $A$ is a $\mu^{\mathscr{V}}$-nowhere dense set in $X$, then the following hold.

(a) If $G$ is a non-null $\mu$ - $\alpha$-open set, then $G \nsubseteq \not A$.

(b) If $H$ is a non-null $\mu$-semi-open set, then $H \nsubseteq A$.

In Theorem 4.9, we replace the condition "wBS-sGTS" by "BS-sGTS" we get $A \in \mu^{\star \star}$, by Corollary 3.2 (b). Theorem 4.9 is not reversible as shown in the following Example 4.12.

Example 4.12. Consider the generalized topological space $\left(X_{5}, \mu\right)$ where $\mu=\{\emptyset,\{a, b\},\{b, c\},\{a, b, c\}\}$. Then $\mu^{\mathscr{V}}=\{\emptyset\} \cup$ $\left\{A \subset X_{5} \mid a \in A\right.$ or $b \in A$ or $\left.c \in A\right\}$.

Let $U=\{c, d\}$. Then $U \in \mu^{\mathscr{V}}$. But $U$ is not a $\mu$ - $\alpha$-open set in $X_{5}$. For, $i_{\mu} c_{\mu} i_{\mu} U=i_{\mu} c_{\mu}(\{\emptyset\})=i_{\mu}(\{d, e\})=\emptyset$. Thus, $U \not \subset i_{\mu} c_{\mu} i_{\mu} U$.

Let $V=\{a, d\}$. Then $V \in \mu^{\mathscr{V}}$. Here $c_{\mu} i_{\mu} V=c_{\mu}(\{\emptyset\})=\{d, e\}$. Thus, $V \not \subset c_{\mu} i_{\mu} V$. Therefore, $V$ is not a $\mu$-semi-open set in $X_{5}$.

Theorem 4.13. Let $(X, \mu)$ be a GTS and $A \subset X$. If $A$ is a $\mu^{\mathscr{V}}$-nowhere dense set, then the following hold.

(a) If $(X, \mu)$ is a wBS, then $A$ is $\mu$-codense.

(b) If $(X, \mu)$ is a sBS, then $A$ is $\mu^{\star}$-codense.

(c) $A$ is $\mu^{\star \star}$-codense set in $X$.

Proof. It is enough to prove (b) only. Suppose $(X, \mu)$ is a sBS and $A$ is a $\mu^{\mathscr{V}}$-nowhere dense set. Then $i_{\mu^{\mathscr{V}}} c_{\mu^{\mathscr{V}}} A=\emptyset$ and so $c_{\mu^{\vartheta}}(X-A)=X$. By hypothesis and Proposition $4.7(\mathrm{~b}), c_{\mu^{\star}}(X-A)=X$. Therefore, $A$ is a $\mu^{\star}$-codense set in $X$.

Proposition 4.14 and Proposition 4.15 are follows from the similar considerations in Lemma 4.3 and Lemma 4.4, respectively so the proofs are omitted.

Proposition 4.14. Let $(X, \mu)$ be $a$ GTS and $A \subset X$. Then

$c_{\mu^{\mathscr{V}}}(A)=\left\{\begin{aligned} X & \text { if } A \text { is } \mu \text {-s-residual, } \\ A & \text { if } A \text { is not } \mu \text {-s-residual }\end{aligned}\right.$

Proposition 4.15. Let $(X, \mu)$ be a GTS, $X$ be a $\mu$-s-II category set and $A \subset X$. Then the following hold.

(a) If $A$ is a $\mu^{\mathscr{V}}$-nowhere dense set, then $A$ is a $\mu$-s-meager set.

(b) If $A$ is a $\mu^{\mathscr{V}}$-meager set, then $A$ is a $\mu$-s-meager set.

(c) If $A$ is a $\mu^{\mathscr{V}}$-residual set, then $A$ is a $\mu$-s-residual set.

(d) If $A$ is of $\mu$-s-II category, then it is of $\mu^{\mathscr{V}}$-II category. 
Theorem 4.16. Let $(X, \mu)$ be a $w B S$. Then every $\mu$-strongly nowhere dense set is $\mu^{\mathscr{V}}$-nowhere dense in $X$.

Proof. Suppose $(X, \mu)$ is a wBS. Let $A$ be a $\mu$-strongly nowhere dense set in $X$. Suppose $i_{\mu^{\gamma}} c_{\mu^{\vee}} A \neq \emptyset$. Then $c_{\mu^{\vee}} A$ contains a non-null $\mu^{\mathscr{V}}$-open set in $X$ and so $c_{\mu^{\mathscr{\gamma}}} A$ contains a $\mu$-s-II category set in $X$. Thus, $c_{\mu} A$ contains a $\mu$-s-II category set in $X$, since $\mu \subset \mu^{\mathscr{V}}$. But $c_{\mu} A$ is a $\mu$-s-meager set in $X$, by Lemma 2.1. Therefore, $i_{\mu^{\mathscr{V}}} c_{\mu^{\gamma}} A=\emptyset$. Hence every $\mu$-strongly nowhere dense set is a $\mu^{\mathscr{V}}$-nowhere dense set in $X$.

The following Corollary 4.17 follows from Theorem 4.16 so the direct proof is omitted.

Corollary 4.17. Let $(X, \mu)$ be a $w B S$ and $A \subset X$. Then the following hold.

(a) If $A$ is $\mu$-s-meager, then $A$ is $\mu^{\mathscr{V}}$-meager in $X$.

(b) If $A$ is of $\mu^{\mathscr{V}}$-II category, then $A$ is of $\mu$-s-II category in $X$.

(c) If $A$ is $\mu$-s-residual, then $A$ is $\mu^{\mathscr{V}}$-residual in $X$.

Theorem 4.18. Let $(X, \mu)$ be a GTS. If $X$ is of $\mu-s-I I$ category, then $\left(X, \mu^{\mathscr{V}}\right)$ is a BS.

Proof. Let $G \in \tilde{\mu^{\mathscr{V}}}$. Suppose $G$ is a $\mu^{\mathscr{V}}$-meager set. Then by hypothesis and Proposition 4.15 (b), $G$ is a $\mu$-s-meager set, which is a contradiction to $G \in \tilde{\mu^{\mathscr{Y}}}$. Therefore, $G$ is of $\mu^{\mathscr{V}}$-II category in $X$. Hence $\left(X, \mu^{\mathscr{V}}\right)$ is a BS.

The following Theorem 4.19 follows from the similar considerations in Theorem 3.19 so the easy proof is omitted.

Theorem 4.19. Let $(X, \mu)$ be a GTS. Then $\mu^{\mathscr{V}} \neq\{\emptyset\}$ if and only if $\mu^{\mathscr{V} \star}$ is a strong GT in $X$ where $\mu^{\mathscr{V}^{\star}}=\left\{\bigcup_{t}\left(W_{1}^{t} \cap W_{2}^{t} \cap \ldots \cap \cap\right.\right.$ $\left.\left.W_{n_{t}}^{t}\right) \mid W_{1}^{t}, W_{2}^{t}, \ldots, W_{n_{t}}^{t} \in \mu^{\mathscr{V}}\right\}$ and hence it is a topology.

In the rest of this section, we give some relations between various types of generalized topology in a generalized topological space.

First of all, we remember some Lemmas which is useful in the sequel.

Lemma 4.20. [14, Theorem 3.4] Let $(X, \mu)$ be a sBS and $A \subset X$. Then the following hold.

(a) If $A$ is a $\mu$-nowhere dense set, then $A$ is a $\mu^{\star}$-nowhere dense set.

(b) If $A$ is a $\mu$-meager set, then $A$ is a $\mu^{\star}$-meager set.

(c) If $A$ is a $\mu^{\star}$-II category set, then $A$ is a $\mu$-II category set.

Lemma 4.21. [14, Theorem 3.7] Let $(X, \mu)$ be a BS and $A \subset X$. Then the following hold.

(a) If $A$ is a $\mu$-nowhere dense set, then $A$ is a $\mu^{\star \star}$-nowhere dense set.

(b) If $A$ is a $\mu$-meager set, then $A$ is a $\mu^{\star \star}$-meager set.

(c) If A is a $\mu^{\star \star}$-II category set, then $A$ is a $\mu$-II category set.

Here, $\mu^{\star \star}=\left\{\bigcup_{t}\left(W_{1}^{t} \cap W_{2}^{t} \cap \ldots \cap \cap W_{n_{t}}^{t}\right) \mid W_{1}^{t}, W_{2}^{t}, \ldots, W_{n_{t}}^{t} \in \mu^{\star \star}\right\}$ and $\mu^{\mathscr{V} \star}=\left\{\bigcup_{t}\left(W_{1}^{t} \cap W_{2}^{t} \cap \ldots \cap W_{n_{t}}^{t}\right) \mid W_{1}^{t}, W_{2}^{t}, \ldots, W_{n_{t}}^{t} \in\right.$ $\left.\mu^{\mathscr{V}}\right\}$.

Now we define two generalized topologies and give some properties of these generalized topologies.

Define $\mu^{\star^{\star \star}}=\{\emptyset\} \cup\left\{A \subset X \mid A\right.$ is of $\mu^{\star}$-II category set $\}$ and $\mu^{\not / \not \star}=\{\emptyset\} \cup\left\{A \subset X \mid A\right.$ is of $\mu^{\mathscr{V}}$-II category set $\}$.

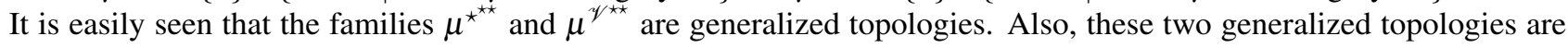
satisfied with the stack property in a GTS.

Theorem 4.22. Let $(X, \mu)$ be a generalized topological space. Then the following hold.

(a) $\tilde{\mu}^{\star \star^{\star}}$ is a stack.

(b) $\tilde{\mu}^{\mathscr{V} \star}$ is a stack.

Proof. It is enough to prove (a) only. Suppose $A \subset B$ where $A \in \tilde{\mu}^{\star \star^{\star}}$. Then $A=\bigcup_{t}\left(A_{1}^{t} \cap A_{2}^{t} \cap \ldots \cap A_{n_{t}}^{t}\right)$ where $A_{1}^{t}, A_{2}^{t}, \ldots, A_{n_{t}}^{t} \in$ $\mu^{\star \star}$. Take $A_{k}=A_{1}^{k} \cap A_{2}^{k} \cap \ldots \cap A_{n_{k}}^{k}$ such that $A_{k} \neq \emptyset$ where $A_{1}^{k}, A_{2}^{k}, \ldots, A_{n_{k}}^{k} \in \mu^{\star \star}$. By hypothesis, $A_{k} \subset B$ so that $B=$ $A_{k} \cup\left(B-A_{k}\right)$. Thus, $B=\left(A_{1}^{k} \cap A_{2}^{k} \cap \ldots \cap \cap A_{n_{k}}^{k}\right) \cup\left(B-A_{k}\right)$ where $A_{1}^{k}, A_{2}^{k}, \ldots, A_{n_{k}}^{k} \in \mu^{\star \star}$ which implies that $B=\left(A_{1}^{k} \cup(B-\right.$ $\left.\left.A_{k}\right)\right) \cap\left(A_{2}^{k} \cup\left(B-A_{k}\right)\right) \cap \ldots \cap\left(A_{n_{k}}^{k} \cup\left(B-A_{k}\right)\right)$ where $A_{1}^{k}, A_{2}^{k}, \ldots, A_{n_{k}}^{k} \in \mu^{\star \star}$. Since $A_{1}^{k}, A_{2}^{k}, \ldots, A_{n_{k}}^{k} \in \tilde{\mu}^{\star \star}$ we have $A_{1}^{k} \cup(B-$ $\left.A_{k}\right), A_{2}^{k} \cup\left(B-A_{k}\right), \ldots, A_{n_{k}}^{k} \cup\left(B-A_{k}\right) \in \tilde{\mu}^{\star \star}$, since $\tilde{\mu}^{\star \star}$ is a stack. Therefore, $B \in \tilde{\mu}^{\star \star^{\star}}$. Hence $\tilde{\mu}^{\star \star^{\star}}$ is a stack.

Corollary 4.23. Let $(X, \mu)$ be a generalized topological space and $\tilde{\mu}$ is a stack. Then $\tilde{\mu}^{\star}$ is a stack.

Obviously, $\mu^{\star \star} \subset \mu^{\star \star^{\star}}$ and $\mu^{\mathscr{V}} \subset \mu^{\mathscr{V} \star}$. The reverse implications are true as shown by the following Theorem 4.24. 
Theorem 4.24. Let $(X, \mu)$ be a generalized topological space. Then the following hold.

(a) If $\left(X, \mu^{\star \star}\right)$ is a sBS, then $\mu^{\star \star} \subset \mu^{\star \star}$.

(b) If $\left(X, \mu^{\mathscr{V}}\right)$ is a sBS, then $\mu^{\mathscr{Y} \star} \subset \mu^{\mathscr{V}}$.

Proof. (a) Suppose $\left(X, \mu^{\star \star}\right)$ is a sBS. Let $G \in \mu^{\star \star}$. If $G=\emptyset$, then there is nothing to prove. Assume that, $G \neq \emptyset$. Then

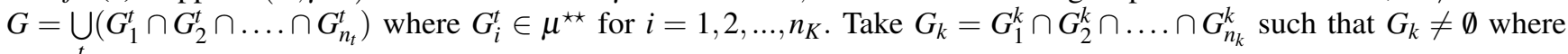
$G_{1}^{k}, G_{2}^{k}, \ldots, G_{n_{k}}^{k} \in \mu^{\star \star}$. By hypothesis, $G_{k}$ is of $\mu^{\star \star}$-II category set in $X$ so that $G_{k}$ is of $\mu$-II category set in $X$, by Lemma 4.21(c). Thus, $G$ is of $\mu$-II category set in $X$. Therefore, $G \in \mu^{\star \star}$. Hence $\mu^{\star \star} \subset \mu^{\star \star}$.

(b) It is follows from the similar arguments in above case and Corollary 4.17 (b).

The condition " $\left(X, \mu^{\mathscr{V}}\right)$ is a sBS" is necessary in Theorem 4.24 (b) as shown by the following Example 4.25

Example 4.25. Consider the generalized topological space $\left(X_{4}, \mu\right)$ where $\mu=\{\emptyset,\{a, b\},\{a, b, c\}\}$. Then $\mu^{\mathscr{V}}=\{\emptyset\} \cup\left\{A \subset X_{4} \mid\right.$ either $a \in A$ or $b \in A\}$. Then $\left(X, \mu^{\mathscr{V}}\right)$ is not a sBS. For, let $U=\{b, d\} ; V=\{a, d\}$. Then $U, V \in \mu^{\mathscr{V}}$. But $U \cap V=\{d\}$ which is a $\mu^{\mathscr{V}}$-nowhere dense set. Here $\mu^{\mathscr{\mathscr { V }}}=\exp (X)$. Thus, $\mu^{\mathscr{V} \star} \nsubseteq \mu^{\mathscr{V}}$.

Theorem 4.26. Let $(X, \mu)$ be a generalized topological space. Then $\mu^{\star \star^{\star}} \subset \mu^{\mathscr{V} \star}$.

Proof. Follows from the fact that $\mu^{\star \star} \subset \mu^{\mathscr{V}}$.

Theorem 4.27. Let $(X, \mu)$ be a wBS. Then $\mu^{\star} \subset \mu^{\mathscr{2} /}$.

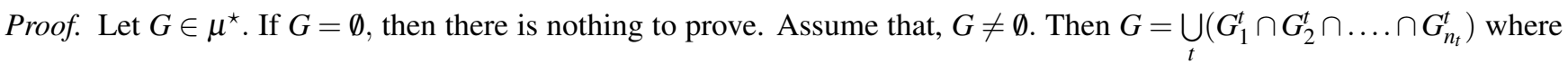
$G_{1}^{t}, G_{2}^{t}, \ldots, G_{n_{t}}^{t} \in \mu$. By hypothesis, $\mu \subset \mu^{\mathscr{V}}$. Thus, $G=\bigcup_{t}\left(G_{1}^{t} \cap G_{2}^{t} \cap \ldots \cap G_{n_{t}}^{t}\right)$ where $G_{1}^{t}, G_{2}^{t}, \ldots, G_{n_{t}}^{t} \in \mu^{\mathscr{V}}$. Therefore, $G \in \mu^{\mathscr{V}^{\star}}$.

Theorem 4.28. Let $(X, \mu)$ be a GTS and $X$ be a $\mu$-s-II category set. Then $\mu^{\mathscr{V}} \subset \mu^{\not / \hbar \star}$.

Proof. Let $B \in \mu^{\mathscr{V}}$. Suppose $B=\emptyset$. Then there is nothing to prove. Assume that, $B \neq \emptyset$. Then $B$ is of $\mu$-s-II category set in $X$. By hypothesis and Proposition 4.15 (d), $B$ is of $\mu^{\mathscr{V}}$-II category set in $X$. Hence $B \in \mu^{\mathscr{V} \star \star}$.

Theorem 4.29. Let $(X, \mu)$ be a sBS. Then the following hold.

(a) $\mu^{\star \star \star} \subset \mu^{\star \star \star}$.

(b) $\mu^{\star \star \star} \subset \mu^{\mathscr{V}}$.

Proof. This follows from Lemma 2.2 and Lemma 4.20.

The following Example 4.30 shows that the reverse implications of Theorem 4.26, Theorem 4.27 and Theorem 4.29 (b) are need not be true in a generalized topological space.

Example 4.30. Consider the generalized topological space $\left(X_{4}, \mu\right)$ where $\mu=\{\emptyset,\{a, b\},\{b, c\},\{a, b, c\}\}$. Then $\mu^{\star}=\{\emptyset,\{b\}$, $\{a, b\},\{b, c\},\{a, b, c\}\} ; \mu^{\star \star}=\{\emptyset\} \cup\{A \subset X \mid b \in A\} ; \mu^{\mathscr{V}}=\{\emptyset\} \cup\{A \subset X \mid a \in A$ or $b \in A$ or $c \in A\} ; \mu^{\star \star}=\{\emptyset\} \cup\{A \subset X \mid$ $b \in A\} ; \mu^{\star \star^{\star}}=\{\emptyset,\{b\},\{a, b\},\{b, c\},\{b, d\},\{a, b, c\},\{a, b, d\},\{b, c, d\}, X\} ; \mu^{\mathscr{V}}=\exp (X)$.

(a). Let $A=\{a\}$. Then $A \in \mu^{\mathscr{V} \star}$. But $A \notin \mu^{\star \star \star}$.

(b). Let $B=\{c\}$. Then $B \in \mu^{\mathscr{V} \star}$. But $B \notin \mu^{\star}$.

(c). Let $C=\{a, d\}$. Then $C \in \mu^{\mathscr{V}}$. But $C \notin \mu^{\star \star \star}$.

The reverse implications of Theorem 4.29 (a) is need not be true as shown by the following Example 4.31.

Example 4.31. Consider the generalized topological space $\left(X_{4}, \mu\right)$ where $\mu=\{\emptyset,\{a\},\{a, b\},\{b, c\},\{a, b, c\}\}$. Then $\mu^{\star}=$ $\{\emptyset,\{a\},\{b\},\{a, b\},\{b, c\},\{a, b, c\}\} ; \mu^{\star \star}=\{\emptyset\} \cup\{A \subset X \mid a \in A$ or $b \in A\}$ and so $\mu^{\star \star \star}=\{\emptyset\} \cup\{B \subset X \mid a \in B$ or $b \in B\} ; \mu^{\star \star}=$ $\exp (X)$. Let $G=\{c\}$. Then $G \in \mu^{\star \star \star}$. But $G \notin \mu^{\star \star \star}$.

Theorem 4.32. Let $(X, \mu)$ be a BS-sGTS. If $\left(X, \mu^{\star \star}\right)$ is a sBS, then $\mu^{\star \star \star} \subset \mu^{\mathscr{V} \star \star}$.

Proof. Let $A$ be a non-null $\mu^{\star \star}$-open set. Then $A=\bigcup_{t}\left(A_{1}^{t} \cap A_{2}^{t} \cap \ldots \cap \cap A_{n_{t}}^{t}\right)$ where $A_{1}^{t}, A_{2}^{t}, \ldots, A_{n_{t}}^{t} \in \mu^{\star \star}$. Take $A_{k}=A_{1}^{k} \cap A_{2}^{k} \cap$ $\ldots \cap \cap A_{n_{k}}^{k}$ where $A_{1}^{k}, A_{2}^{k}, \ldots, A_{n_{k}}^{k} \in \mu^{\star \star}$ such that $A_{k} \neq \emptyset$ for some $k$. By hypothesis, $A_{k}$ is of $\mu^{\star \star}$-II category set and so $A_{k}$ is of $\mu$-II category set, by hypothesis and Lemma 4.21. Thus, $A$ is of $\mu$-II category set so that $A$ is of $\mu$-s-II category set. Hence $A \in \mu^{\mathscr{V}}$. By Theorem 4.28, $A \in \mu^{\mathscr{V}^{\star \star}}$. Hence $\mu^{\star \star \star} \subset \mu^{\mathscr{V}^{\star \star}}$. 


\section{Some Special Spaces}

In this section, we analyze the nature of extremally disconnected and submaximal spaces in a generalized topological space. Finally, we prove every $\mu$-isolated point is a $\mu$-II category set in a GTS.

A GTS $(X, \mu)$ is called $\mu$-extremally disconnected or simply, extremally-disconnected [4] if the $\mu$-closure of every $\mu$-open set is $\mu$-open.

A subset $B$ of a generalized topological space $(X, \mu)$ is said to be a $\mu$ - $G_{\delta}$-set [1] if $B=\bigcap_{n \in \mathbb{N}} B_{n}$ where each $B_{n}$ is a $\mu$-open set.

A generalized topological space $(X, \mu)$ is said to be a generalized $G_{\delta}$-submaximal space [1] if every $\mu$-dense subset of $X$ is a $\mu-G_{\delta}$-set in $X$.

Lemma 5.1. [1, Lemma 3.7] Let $(X, \mu)$ be a GTS. If $(X, \mu)$ is a generalized submaximal space, then $(X, \mu)$ is a generalized $G_{\delta}$-submaximal space.

Lemma 5.2. [13, Theorem 3.2] Let $(X, \mu)$ be a GTS. Then the following hold.

(a) $\mu^{\star \star} \neq\{\emptyset\}$ if and only if $\left(X, \mu^{\star \star}\right)$ is a sGTS.

(b) If $(X, \mu)$ is a $B S$, then $\mu^{\star \star} \neq\{\emptyset\}$.

Theorem 5.3. Let $(X, \mu)$ be a GTS. If either $(X, \mu)$ is a BS or $\mu^{\star \star} \neq\{\emptyset\}$, then $\left(X, \mu^{\star \star}\right)$ is a $\mu^{\star \star}$-extremally disconnected space.

Proof. We will present the detailed proof only for the case, $\mu^{\star \star} \neq\{\emptyset\}$. Then $\mu^{\star \star}$ is a sGTS, by Lemma 5.2. Let $G \in \mu^{\star \star}$. If $G=\emptyset$, then $c_{\mu^{\star \star}} G=G$ and so $c_{\mu^{\star \star}} G \in \mu^{\star \star}$. Suppose that $G \neq \emptyset$. Then $G$ is of $\mu$-II category set in $X$. Since $G \subset c_{\mu^{\star \star}} G$ and subset of a $\mu$-meager set is $\mu$-meager we have $c_{\mu^{\star}} G$ is of $\mu$-II category set in $X$. Thus, $c_{\mu^{\star \star}} G \in \mu^{\star \star}$. Hence $\left(X, \mu^{\star \star}\right)$ is a $\mu^{\star \star}$-extremally disconnected space.

The following Example 5.4 shows that the condition "either $(X, \mu)$ is a Baire space or $\mu^{\star \star} \neq\{\emptyset\}$ " can not be dropped in Theorem 5.3.

Example 5.4. Consider the generalized topological space $(X, \mu)$ where $X=[0,3]$ and $\mu=\{\emptyset,[0,2),(1,3],[0,1] \cup[2,3], X\}$. Then $(X, \mu)$ is not a BS and $\mu^{\star \star}=\{\emptyset\}$. Choose $G \in \mu^{\star \star}$. Then $G=\emptyset$ and so $c_{\mu^{\star \star}} G=X$. But $X \notin \mu^{\star \star}$. Thus, $c_{\mu^{\star \star}} G \notin \mu^{\star \star}$. Hence $\left(X, \mu^{\star \star}\right)$ is not a $\mu^{\star \star}$-extremally disconnected space.

Theorem 5.5. Let $(X, \mu)$ be a GTS. Then the following hold.

(a) If $\tilde{\mu}$ is a stack, then $(X, \mu)$ is a $\mu$-extremally disconnected space.

(b) If $\tilde{\mu}$ is a p-stack, then $(X, \mu)$ is a $\mu$-extremally disconnected space.

Proof. It is enough to prove that (a) only, since every p-stack is a stack. Suppose that, $\tilde{\mu}$ is a stack. Then $(X, \mu)$ is a sGTS. Let $U \in \mu$. If $U=\emptyset$, then $c_{\mu} U=\emptyset$, since $\mu$ is a sGT. Thus, $c_{\mu} U \in \mu$. Assume that, $U \neq \emptyset$. Since $U \subset c_{\mu} U$ and $\tilde{\mu}$ is a stack we have $c_{\mu} U \in \mu$. Then $(X, \mu)$ is a $\mu$-extremally disconnected space.

Next, Example 5.6 shows that the condition " $\tilde{\mu}$ is a stack" can not be dropped in the above Theorem 5.5 (a). The reverse implications of Theorem 5.5 is need not be true as shown by the below Example 5.7.

Example 5.6. (a) Consider the generalized topological space $(X, \mu)$ where $X=[0,3]$ and $\mu=\{\emptyset,[0,1),[0,2),(1,3],[0,1) \cup$ $\left.(1,3],[0,2) \cup\left[\frac{5}{2}, 3\right], X\right\}$. Let $A=(1,3]$ and $B=[1,3]$ be subsets of $X$. Here $A \in \tilde{\mu}$ and $A \subset B$. But $B \notin \tilde{\mu}$. Thus, $\tilde{\mu}$ is not a stack. Take $G=[0,1)$. Then $G \in \mu$ and $c_{\mu} G=[0,1]$. But $c_{\mu} G \notin \mu$. Hence $(X, \mu)$ is not a $\mu$-extremally disconnected space.

(b) Consider the generalized topological space $\left(X_{6}, \mu\right)$ where $\mu=\{\emptyset,\{a, b\},\{b, c\},\{a, b, c\}\}$. Let $A=\{a, b\}$ and $B=\{a, b, c, d\}$ be subsets of $X_{6}$. Here $A \in \tilde{\mu}$ and $A \subset B$. But $B \notin \tilde{\mu}$. Thus, $\tilde{\mu}$ is not a stack. Take $G=\{\emptyset\}$. Then $G \in \mu$ and $c_{\mu} G=\{d, e, f\}$. But $c_{\mu} G \notin \mu$. Hence $\left(X_{6}, \mu\right)$ is not a $\mu$-extremally disconnected space.

Example 5.7. (a) Consider the generalized topological space $\left(X_{5}, \mu\right)$ where $\mu=\left\{\emptyset,\{a, c\},\{b, c\},\{a, b, c\}, X_{5}\right\}$. Then $\left(X_{5}, \mu\right)$ is a $\mu$-extremally disconnected space. Let $A=\{a, c\}$ and $B=\{a, b, c, d\}$ be subsets of $X_{5}$. Here $A \in \tilde{\mu}$ and $A \subset B$. But $B \notin \tilde{\mu}$. Thus, $\tilde{\mu}$ is not a stack.

(b) Consider the generalized topological space $\left(X_{4}, \mu\right)$ where $\mu=\{\emptyset,\{a\},\{b\},\{a, b\},\{a, c\},\{a, d\},\{b, c\},\{b, d\},\{a, b, c\},\{a, b, d\}$, $\left.\{a, c, d\},\{b, c, d\}, X_{4}\right\}$. Then $\left(X_{4}, \mu\right)$ is a $\mu$-extremally disconnected space. But $\tilde{\mu}$ is not a p-stack. For, let $A=\{a\}$ and $B=\{b, c, d\}$ be subsets of $X_{4}$. Here $A \in \tilde{\mu}$ and $B \in \tilde{\mu}$. But $A \cap B=\emptyset$.

The following Theorem 5.8 is directly follows from the fact that subset of a $\mu$-s-meager set is $\mu$-s-meager and the converse part is trivial so the proof is omitted. 
Theorem 5.8. Let $(X, \mu)$ be a GTS. Then $\mu^{\mathscr{V}} \neq\{\emptyset\}$ if and only if $\left(X, \mu^{\mathscr{V}}\right)$ is a $\mu^{\mathscr{V}}$-extremally disconnected space.

Theorem 5.9. Let $(X, \mu)$ be a hyperconnected sGTS. Then $(X, \mu)$ is a $\mu$-extremally disconnected space.

Proof. This is a direct consequence of the definition of the hyperconnected space.

By Lemma 2.1, immediately we get the following two observations so the proofs are omitted.

Observation 5.10. Let $(X, \mu)$ be a GTS and $\mu=\{\emptyset\} \cup\{A \subset X \mid A$ is a $\mu$-nowhere dense set $\}$. If $\mu$ is a GT, then $(X, \mu)$ is a $\mu$-extremally disconnected space.

Observation 5.11. Let $(X, \mu)$ be $a$ GTS and $\mu=\{\emptyset\} \cup\{A \subset X \mid A$ is a $\mu$-strongly nowhere dense set $\}$. If $\mu$ is $a$ GT, then $(X, \mu)$ is a $\mu$-extremally disconnected space.

Moreover, every GTS $(X, \mu)$ is both $\mu^{\star \star}$-extremally disconnected space and $\mu^{\mathscr{V}}$-extremally disconnected space.

Lemma 5.12. Let $(X, \mu)$ be a GTS and $A \subset X$. Then the following hold.

(a) If $A$ is a $\mu^{\star \star}$-dense set in $X$, then $A \in \mu^{\star \star}$.

(b) If $A$ is a $\mu^{\mathscr{V}}$-dense set in $X$, then $A \in \mu^{\mathscr{V}}$.

Proof. We will present the detailed proof only for (a). Let $A$ be a $\mu^{\star \star}$-dense subset of $X$. Then $A \cap B_{i} \neq \emptyset$ for all $B_{i} \in \tilde{\mu}^{\star \star}$.

Case 1: First we prove this result for a singleton set in $\tilde{\mu}^{\star \star}$. Assume that, each $C_{i}$ is a singleton set in $\tilde{\mu}^{\star \star}$. Since $A \cap C_{i} \neq \emptyset$ for all $C_{i} \in \tilde{\mu}^{\star \star}$ we have $C_{i} \subset A$ for all $C_{i} \in \tilde{\mu}^{\star \star}$. Therefore, $A$ is of $\mu$-II category set in $X$, since subset of a $\mu$-meager set is $\mu$-meager.

Case 2: Now we prove this result for other set in $\tilde{\mu}^{\star \star}$. Assume that, each $B_{i}$ having more than one element. Then each $B_{i}$ contains a non-null singleton set which is of $\mu$-II category set in $X$. By Case $1, A$ is of $\mu$-II category set in $X$.

The reverse implication of Lemma 5.12 need not be true as shown by Example 5.13.

Example 5.13. (a) Consider the generalized topological space $(X, \mu)$ where $X=[0,3]$ and $\mu=\{\emptyset,[0,2),(1,3],[0,1) \cup$ $\left.(1,3],[0,2) \cup\left[\frac{5}{2}, 3\right], X\right\}$. Then $\mu^{\star \star}=\{\emptyset\} \cup\{A, B \subset X \mid A \in \exp ((1,2))-\{\emptyset\}, A \subset B\}$. Let $A=\left\{\frac{3}{2}\right\}$ be a subset of $X$. Then $A \in \mu^{\star \star}$. But $A$ is not a $\mu^{\star \star}$-dense set in $X$.

(b) Consider the generalized topological space $\left(X_{6}, \mu\right)$ where $\mu=\{\emptyset,\{a, b\},\{a, b, c\},\{a, b, d\},\{a, b, c, d\},\{a, b, c, e\}\{a, b, c, d, e\}\}$. Then $\mu^{\mathscr{V}}=\{\emptyset\} \cup\left\{A \subset X_{6} \mid\right.$ either $a \in A$ or $\left.b \in A\right\}$. Let $G=\{a, d\}$ be a subset of $X_{6}$. Then $G \in \mu^{\mathscr{V}}$. But $G$ is not a $\mu^{\mathscr{V}}$-dense set in $X_{6}$.

Theorem 5.14. Let $(X, \mu)$ be a GTS. Then $\mu^{\mathscr{V}} \neq\{\emptyset\}$ if and only if $\left(X, \mu^{\mathscr{V}}\right)$ is a generalized submaximal space.

Proof. Let $A$ be a $\mu^{\mathscr{V}}$-dense set in $X$. Then $A$ is a $\mu^{\mathscr{V}}$-open set in $X$, by Lemma 5.12(b). Therefore, $\left(X, \mu^{\mathscr{V}}\right)$ is a generalized submaximal space. Converse implication is trivial.

Corollary 5.15 is directly follows from Lemma 5.1 and Theorem 5.14 so the proof is omitted.

Corollary 5.15. Let $(X, \mu)$ be a GTS. Then $\mu^{\mathscr{V}} \neq\{\emptyset\}$ if and only if $\left(X, \mu^{\mathscr{V}}\right)$ is a generalized $G_{\delta^{-}}$submaximal space.

Theorem 5.16. Let $(X, \mu)$ be a GTS. Then $\mu^{\star \star} \neq\{\emptyset\}$ if and only if $\left(X, \mu^{\star \star}\right)$ is a generalized submaximal space.

Corollary 5.17. Let $(X, \mu)$ be a GTS. Then $\mu^{\star \star} \neq\{\emptyset\}$ if and only if $\left(X, \mu^{\star \star}\right)$ is a generalized $G_{\delta}$-submaximal space.

In the rest of this section, we analyze the nature of an isolated point in a GTS. First of all, we remind the definition for isolated point in a generalized topological space.

Let $(X, \mu)$ be a generalized topological space. Then $x \in X$ is called $\mu$-isolated [1] if $\{x\}$ is $\mu$-open. If every point of $X$ is $\mu$-isolated, then $X$ is called $\mu$-discrete [1].

Theorem 5.18. Let $(X, \mu)$ be a GTS and $x \in X$. If $x$ is a $\mu$-isolated point, then the following hold.

(a) $\{x\} \in \mu^{\star \star}$.

(b) $\{x\} \in \mu^{\mathscr{V}}$.

(c) $X$ is of $\mu$-II category.

(d) $X$ is of $\mu$-s-II category. 
Proof. (a) Let $x \in X$. Suppose $x$ is a $\mu$-isolated point in $X$. Take $A=\{x\}$. Then $A$ is a $\mu$-open subset of $X$ and so $A$ is not a $\mu$-nowhere dense set. Thus, $A$ is not a $\mu$-meager set so that $A$ is of $\mu$-II category set in $X$. Therefore, $\{x\} \in \mu^{\star \star}$.

(b) Since $\mu^{\star \star} \subset \mu^{\mathscr{V}}$ we have $\{x\} \in \mu^{\mathscr{V}}$, by (a).

(c) Superset of a $\mu$-II category set is of $\mu$-II category so that $X$ is of $\mu$-II category.

(d) Since every $\mu$-s-meager set is $\mu$-meager we have $X$ is of $\mu$-s-II category.

Theorem 5.19 immediately follows from Theorem 5.18 so the trivial proof is removed. The reverse implications of the Theorem 5.19 is not true in general as shown in the below Example 5.20.

Theorem 5.19. Let $(X, \mu)$ be $a$ GTS and $A \subset X$. Then the following hold.

(a) If $X$ is $\mu$-discrete, then $(X, \mu)$ is a sBS.

(b) If $A$ contains a $\mu$-isolated point, then $A \in \mu^{\star \star}$ and hence $A \in \mu^{\mathscr{V}}$.

Example 5.20. Consider the generalized topological space $\left(X_{4}, \mu\right)$ where $\mu=\left\{\emptyset,\{a, b\},\{b, c\},\{a, b, c\}, X_{4}\right\}$. Then $\left(X_{4}, \mu\right)$ is a sBS. But $X_{4}$ is not a $\mu$-discrete space. For, let $a \in X_{4}$. Then $\{a\}$ is not $\mu$-open and so $a$ is not a $\mu$-isolated point in $X_{4}$.

Theorem 5.21. Let $(X, \mu)$ be a GTS and $A \subset X$. Then $A$ is of $\mu$-II category set in $X$ if and only if it has a $\mu^{\star \star-i s o l a t e d ~ p o i n t ~ i n ~}$ $X$.

The following Example 5.22 proves that $X$ is not $\mu$-discrete even if $X$ is $\mu^{\star \star}$-discrete.

Example 5.22. Consider the generalized topological space $\left(X_{4}, \mu\right)$ where $\mu=\left\{\emptyset,\{a, b\},\{c, d\}, X_{4}\right\}$. Here $\{x\} \in \mu^{\star \star}$ for all $x \in X_{4}$. Therefore, $X_{4}$ is $\mu^{\star \star}$-discrete. But $X_{4}$ is not a $\mu$-discrete space. For, let $b \in X_{4}$. Then $\{b\}$ is not $\mu$-open and so $b$ is not a $\mu$-isolated point in $X_{4}$.

Lemma 5.23. [14, Theorem 4.3] Let $(X, \mu)$ be a hyperconnected space. If $X$ is of $\mu$-II category, then $(X, \mu)$ is a BS.

Theorem 5.24. Let $(X, \mu)$ be a GTS and $\tilde{\mu}$ is a p-stack. If $X$ is of $\mu$-II category, then $(X, \mu)$ is a BS.

Proof. Suppose $\tilde{\mu}$ is a p-stack. Then $(X, \mu)$ is a hyperconnected space. By Lemma $5.23,(X, \mu)$ is a BS.

\section{References}

[1] M. R. Ahmadi Zand and R. Khayyeri, Generalized $G_{\delta}$-submaximal spaces, Acta Math. Hungar., 149 (2) (2016), 274 - 285.

[2] S. Al Ghour, A. Al-Omari and T. Noiri, On homogeneity and homogeneity components in generalized topological spaces, Filomat, 27 (2013), 1097 - 1105.

[3] Á. Császár, Generalized open sets, Acta Math. Hungar., 75 (1997), 65 - 87.

[4] Á. Császár, Extremally disconnected generalized topologies, Annales Univ. Sci. Budapest., 47 (2004), 91 - 96.

[5] Á. Császár, Generalized open sets in generalized topologies, Acta Math. Hungar. 106 (1 - 2) (2005), 53 - 66.

[6] E. Ekici, Generalized hyperconnectedness, Acta Mathematica Hungarica, 133 (1 - 2) (2011), 140 - 147.

[7] E. Ekici, Generalized Submaximal Spaces, Acta Math. Hungar., 134 (1 - 2) (2012), 132 - 138.

[8] E. Korczak - Kubiak, A. Loranty and R. J. Pawlak, Baire generalized topological spaces, generalized metric spaces and infinite games, Acta Math. Hungar., 140 (2013), 203 - 231.

[9] Z. Li and F. Lin, Baireness on generalized topological spaces, Acta Math. Hungar., 139 (4) (2013), 320 - 336.

[10] W. K. Min, On weak neighborhood systems and spaces, Acta Math. Hungar., 121 (3) (2008), 283 - 292.

[11] V. Renukadevi and S. Vadakasi, On lower and upper semi-continuous functions, Acta Math. Hungar., 160 (2020), 1 - 12.

[12] S. Vadakasi and V. Renukadevi, Properties of nowhere dense sets in GTSs, Kyungpook Math. J., 57 (2017), 199 - 210.

[13] S. Vadakasi and V. Renukadevi, Special functions on GTSs, Communicated.

[14] S. Vadakasi and V. Renukadevi Two classes of functions, Communicated. 